\section{Is periodontal disease a public health problem?}

P. Batchelor ${ }^{1}$

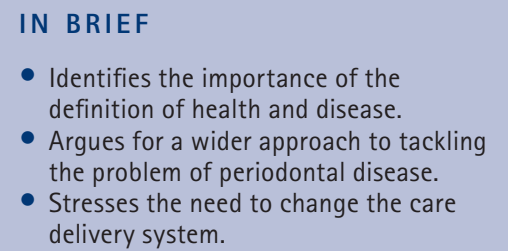

Clinically defined periodontal disease is highly prevalent, has considerable impacts on individuals and society and is costly to treat; the cost of dental care is the fourth highest costs of all diseases and consuming between 5 and 10\% of all healthcare resources. Changes in the epidemiology of clinically defined periodontal diseases suggest that the prevalence of severe periodontal disease is low and rates of progression of periodontal destruction tend to be relatively slow. Current periodontal care modalities have a remarkably weak evidence base, with considerable resources allocated to fund interventions that include oral hygiene instruction, scale and polishes through to surgical interventions. The public health problem lies more in the failure in design of a contract between dental professionals and the state. Such a contract needs to recognise both the wider determinants of disease and the role that dental professionals could play: a contract that concentrated on rewarding outcomes, namely a diminution in treatment need, as opposed to one based simply on the number of interventions would be a major step forward.

\section{INTRODUCTION}

Paedersen $^{1}$ in a global review of oral diseases highlighted how oral diseases qualified as major public health problems due to their high prevalence and incidence in all regions of the world. He went on to suggest that approaches to managing the problems ranged from intensive one-to-one relationships between the patient and the care provider through to a 'public health approach'. The balance between the two would be determined by a number of factors including the distribution of the condition and available resources. Defining how a condition is best managed is critical for a number of reasons. Most importantly, with the increasing financial pressure on healthcare systems, there is a need to ensure that for a given outcome, expenditure is minimised; the cost effectiveness of differing interventions is key in decision making.

There are a number of criteria that are used to establish whether a condition is a public health problem ${ }^{2}$ and consequently appropriate to be managed using a public

'Dental Public Health Unit, Department of Epidemiology and Public Health, University College London,

119 Torrington Place, London, WC1E 6BT

Correspondence to: Dr Paul Batchelor

Email: p.batchelor@ucl.ac.uk

\section{Refereed Paper}

Accepted 8 August 2014

DOI: 10.1038/sj.bdj.2014.912

${ }^{\circledR}$ British Dental Journal 2014; 217: 405-409 health approach. Periodontal disease fulfils them: it is widespread; its consequences in terms of social, psychological and economic impacts on individuals, communities and health services are severe; the costs to society and to individuals are considerable; and effective methods are available to prevent, alleviate or cure the disease. Given the above, why then does periodontal disease remain such a problem?

This article will explore this question and suggest that the extremes of periodontal disease are limited although for those that do suffer, it impacts severely on their qualities of life. The manifestations of periodontitis - bleeding, halitosis, gingival recession and tooth loss - can also have an impact beyond the individual sufferer. The costs of treating the disease are high because of the way in which dental care is organised: the costs of treatment are expensive for individuals and societies and remain so due to the current inappropriate approaches used to manage the conditions.

The current state of knowledge of the diseases, and in particular associated risk factors, means that while there is sufficient information to enable control of the common forms of the disease, existing interventions are not effective.

The key conclusion is that until shortcomings in currently adopted practices at all levels, including those at societal and within the care system are addressed, periodontal disease will remain a public health problem. The challenge is to recognise the key issues: both politicians and dental professions, as advocates for patients, need to act to address them. Health is a political problem and only when the design of the delivery system allows care workers to act as advocates for the public will the problems of periodontal disease be tackled.

\section{DEFINING A PUBLIC HEALTH PROBLEM}

Defining a public health problem forms the central argument to the question as to whether a health issue is a 'public health' as opposed to simply a 'health' problem. The distinction lies in what is understood by the term public health. Winslow ${ }^{3}$ argued that public health was: "the science and art of preventing disease, prolonging life and promoting health through the organized efforts and informed choices of society, organizations, public and private, communities and individuals'

Over 80 years later Rothstein ${ }^{4}$ reiterated the general sentiments in the definition arguing that there were three issues to distinguish between public health and health problems. These were where the health of the population is threatened by something (including environmental factors not just diseases); where the government has powers or expertise to meet that threat; and where the action of government will be more 
efficient or more likely to be beneficial than the actions of individuals.

This definition recognises that if a problem exists, the solution is not simply derived through individual actions, for example attending for a dental appointment, but by those of society. However, this still fails to address whether any given condition is a problem. Sheiham ${ }^{5}$ suggested a number of criteria that are necessary to help establish whether the issue under consideration is indeed a public health problem. The criteria include: the distribution and extent of the condition, namely how widespread is it and whether the prevalence is increasing or decreasing; and the impact of the condition on individuals, for example the extent to which the condition causes pain, discomfort and affects functions such as eating, speaking, sleeping and social interactions that cause embarrassment. Further impacts would include the financial cost of treatment, absence from work and loss of income that in turn may lead to impacts on the wider community. This would include the effect it may have on people attending school or work. There are the costs to the health services of treating the condition and finally, and most importantly, is the condition preventable and are effective treatments available?

Periodontal disease fulfils all the criteria. As Chapple has stated recently: 'Periodontitis is the most common chronic inflammatory disease seen in humans, affecting nearly half of adults in the United Kingdom and $60 \%$ of those over 65 years. It is a major public health problem, causing tooth loss, disability, masticatory dysfunction, and poor nutritional status. Periodontitis also compromises speech, reduces quality of life, and is an escalating burden to the healthcare economy. ${ }^{6}$

In summary, periodontal disease using a disease definition is highly prevalent, has considerable impacts on individuals and society and is costly to treat; the cost of dental care is the fourth highest costs of all diseases and consuming between 5 and 10\% of all healthcare resources..$^{7-8}$ Periodontal diseases are in the vast majority preventable and there are effective methods of managing them.

\section{THE NATURE OF \\ PERIODONTAL DISEASE}

Our understanding of the disease process itself has changed considerably over the years. Sheiham ${ }^{6}$ has summarised the changes as follows. At the population level the prevalence of destructive periodontal disease is considerably lower than previously estimated and, in most industrialised countries, the public's periodontal health appears to be improving. Internationally, the prevalence of severe periodontal disease is low and rates of progression of periodontal destruction tend to be relatively slow. A small proportion of subjects exhibit severe and extensive periodontitis with approximately 1 in 1,000 suffering from aggressive periodontitis. Mild gingivitis is common in children and adults, and very few children demonstrate loss of bony support and loss of periodontal attachment. There is consensus that severe periodontal disease occurs in a few teeth in a relatively small proportion of people in any given age group, and that the proportion affected is greater in older age-groups.

At an individual level there have also been considerable changes in our understanding of the disease with the natural history of periodontal disease progression being more complex than previously suggested. The old 'continuous progression' model of periodontal disease considers that gingivitis progress to periodontitis. A slow loss of attachment follows and its bony support progressing continuously until the tooth is non-functional. Such a model suggests that once a person has periodontal disease only continuous treatment will prevent the inevitable progress of the destructive lesion to severe periodontitis. There is little evidence to support this model. More recently, the model described has been challenged by one based on 'bursts'. Key differences are recognising that not all gingivitis progresses to irreversible periodontitis and that not all mild periodontitis progresses to severe periodontitis. Individuals exhibit differences in exposure and resistance and the most serious phases of periodontitis being not as common as formerly thought. Finally, there are growing questions about the extent to which periodontal disease causes tooth loss. ${ }^{9}$

These developments, however, do not detract from periodontal disease being a public health problem, but highlight that it is essentially socio-political in character. As with other major non-communicable diseases such as cardiovascular disease, obesity, diabetes and cancers, the social determinants of dental diseases share common antecedents. ${ }^{10-12}$ Significant control of dental diseases can only be achieved in terms of social policy. The task of health workers is to convince society to undertake the specific social measures that are required to solve health problems, and to participate in the implementation of these policies. Avoiding the need for developing effective social policies for health in favour of concentration on problems of individual health behaviour is not only oversimplification but an evasion of professional responsibility.

\section{FINDING AN APPROPRIATE SOLUTION}

All societies aim to ensure the health of their citizens. Governments try and achieve this by developing healthcare systems that set out to address three main challenges: how to improve the health status of both individuals and the population; how to develop arrangements to protect citizens from threats to their health and the costs of care should they require it; and how to ensure equitable access. ${ }^{13}$ The contextual setting for the above challenges that all care systems face is that of limited resources. Even the wealthiest societies face limitations in what care they can provide. All healthcare delivery systems have implicit or explicit boundaries on the availability of care related to whether a society limits specific types of interventions for all citizens, limits the occasions when an individual can receive it or simply who can receive it.

These issues raise the further question on the arrangements surrounding the decisionmaking processes within a system. Is the design of the system aimed at ensuring those who seek care can have it or is there an emphasis on targeting those with the greater needs? How is the balance between the more immediate interventions that address a problem, for example dealing with acute myocardial infarctions, balanced against longer term issues such as preventing heart disease in the first place? What is the process to decide on allocating spending on say cancer rather than dental care? Such questions have led to the necessity to identify mechanisms to prioritise, not only which interventions a society is willing to provide for its citizens, but also the arrangements through which the care will be delivered. That is far from simple. Musgrove ${ }^{14}$ highlighted nine criteria grouped under three themes for helping identify issues when considering the justification for public spending on healthcare. These range from economic efficiency, ethical reasons and political considerations. Referring to a previous authored paper, Musgrove ${ }^{15}$ added: '[Simply] being a public good is not reason enough for the government to finance a healthcare intervention, because the result in improved health might not be worth the cost - the same resources could be better used for another health service or for some non-health activity.'

The issues raised by Musgrove get to the heart of the matter to help establish whether a health condition is worth addressing or to consider if there are benefits that might be accrued through other arrangements. Musgrove recognises that the funding of interventions outside of the healthcare system may well provide improvements in health conditions. 
The nature of health problems facing care systems has seen the balance between acute and chronic diseases change. Currently, it is the management of chronic disease that set the challenges: obesity, diabetes, respiratory and coronary conditions. Societies are attempting to adapt their care systems to confront the challenges, not least by adopting a growing role for what is termed a 'public health' approach. Such an approach introduces the idea of identifying the 'cause of the cause' and even the 'cause of the cause of the cause. ${ }^{16}$ It makes the important distinction between disease and the determinants of disease.

The outstanding issue of what we actually mean by health remains. No longer is a definition based simply the absence of clinical disease adequate. The term has a wider meaning and needs to include various social dimensions, for example function and well-being.

\section{THE CRITICAL ISSUE OF DEFINING PERIODONTAL DISEASE AND ITS NATURAL HISTORY}

As discussed earlier our understanding of the nature and epidemiology of conditions affecting the periodontal tissues has changed considerably over the last 50 years. ${ }^{17}$ They identify four main features of what they termed the periodontal diseases. These are that: clinical attachment loss of $1 \mathrm{~mm}$ or greater is highly prevalent even in very young subjects; within a given population, the prevalence of attachment loss, the extent of attachment loss and the severity of attachment loss increase with age; within a given age group, the distribution of the extent and severity of destruction tends to be right-skewed to such a degree that a minor fraction of the subjects carries the major burden of destruction in the group; and, within a given population, the intra-oral pattern of distribution of periodontal destruction is rather distinct and corroborates the molarincisor pattern originally considered characteristic for juvenile periodontitis.

What Baelum and Lopez ${ }^{18}$ highlight is how the definitions of periodontal disease have altered to meet the requirements of a particular set of scientific beliefs. The definitions have changed from a time when a microbiological solution was sought: 'plaque-induced' and 'not plaque-induced' periodontal disease, through a period when 'active' and 'inactive' sites might provide an insight into addressing the professionally defined problem, to the current vogue of 'periodontal medicine', in which an individual's general health is linked to their periodontal status.

These issues of definition of what is periodontal disease are critical. As Baelum and
Lopez state, the distinction between what is termed periodontal health and disease is not fixed. The definitions change according to professional agendas. This is a view that is in agreement with Borrell and Papapanou ${ }^{19}$ who highlighted the lack of uniformity in definitions and highlights a key failing of current care delivery systems. The systems concentrate on a 'disease' model and pay no heed to the wider definitions of health. Because a person may have a number of teeth with a loss of attachment of 4 or $5 \mathrm{~mm}$ is in itself not a problem. The real issue is how the periodontal state impacts on the qualities of life of individuals.

What such a broader approach creates is a problem when assessing the magnitude of future health problems. Individuals are now retaining more teeth and for longer. This has a direct impact on the size of the periodontal problem. For example, an individual who has lost all their teeth does not have a periodontal problem. However if they retain more teeth that are functional and in which the aesthetics are acceptable, the volume of the problem will still increase if a disease-based model is used to quantify the magnitude of the problem. Unless the assessment is made in terms of impacts on qualities of life, the additional number of pockets can only create a higher level of clinically assessed disease.

The above issues highlight the importance of defining what periodontal disease is. It is not the level of disease per se, but its definition that is critical when answering the question as to whether periodontal disease is a public health problem.

\section{HOW EFFECTIVE ARE CURRENT CARE MODALITIES?}

Notwithstanding the lack of definitions there still remains a need to assess the impact of care modalities. Considerable resources are allocated to fund interventions that include oral hygiene instruction, scale and polishes through to surgical interventions. These have a remarkably weak evidence base. Perhaps the most damning of statements regarding progress in identifying appropriate care modalities for periodontal disease was that provided by Herrera et al. ${ }^{19}$ They felt that: it would be inappropriate to make definitive and specific recommendations regarding clinical practice based on the limited metaanalysis and the review of these 25 studies'.

Needleman et $a .^{20}$ in a systematic review of professional mechanical plaque removal for prevention of periodontal diseases also drew similar conclusions regarding the lack of evidence to underpin clinical practice. They stated: 'There appears to be little value in providing professional mechanical plaque removal (PMPR) without oral hygiene instruction (OHI). In fact, repeated OHI might have a similar effect as PMPR. Some forms of PMPR might achieve greater patient satisfaction. There is little difference in beneficial or adverse effects of different methods of PMPR.'

Bierne et $a l .{ }^{21}$ also reinforced this view. They concluded: '(the evidence) is of insufficient quality to reach any conclusions regarding the beneficial and adverse effects of routine scaling and polishing for periodontal health and regarding the effects of providing this intervention at different time intervals.'

Perhaps the most critical observation was that made by Sheiham and Netuveli ${ }^{22}$ who stated: 'In an era of evidence-based healthcare, the current uncritical position where any inflammation of the gingivae or shallow pocketing is considered in need of treatment is untenable. Advanced periodontal disease does affect a relatively small percentage of adults and is more common in older people. The progression pattern of the disease seems compatible with retention of a functional dentition throughout life for the majority of people in Europe.'

All the authors of reviews come to remarkably similar conclusions. The evidence base to underpin care modalities is very poor. There is not only a lack of consensus in what is termed periodontal disease but the care modalities themselves lack justification for their adoption. This indicates that if a problem in managing periodontal health does exist it may be far more deep-rooted than the disease itself.

\section{SO WHAT EXACTLY IS THE PROBLEM?}

Hugoson and Norderyd ${ }^{23}$ provided a review of global trends in the prevalence of periodontitis. Using the existing clinical indices they concluded that there was a strong possibility of a trend of a lower prevalence especially within Europe and the USA with more specific data from Sweden showing that periodontal health can be significantly improved at the population level. The question that arises is how is this being achieved?

A theoretical framework for improving periodontal health that recognises the wider determinants has been proposed by Watt and Petersen. ${ }^{30}$ In their review they concluded that periodontal diseases affect a significant proportion of the world's population, particularly the socially disadvantaged. They added that inequalities in periodontal health mirror those patterns in other chronic diseases and share common determinants and concluded that clinical treatment and chairside preventive advice alone will never tackle the problem. 
A mechanism to overcome the shortfalls was proposed by Thomson et al..$^{25}$ They provided a coherent approach to addressing the problems that periodontal disease creates based on three strategies: (i) a population strategy for altering life practices, particularly those determining smoking behaviour and oral self-care (plaque removal) in the community; (ii) a secondary prevention strategy to detect and treat people with destructive periodontal disease; and (iii) a high-risk strategy for treating existing disease and preventing further disease in those at special risk. They argued that the Common Risk Factor Approach and the health promotion approach are key aspects of these strategies.

Indeed, Lopez and Baelum ${ }^{26}$ reinforced the need to step outside of the traditional care system arguing some of the interventions that might be relevant for the prevention and control of periodontitis at the population level do not fall within the territories of traditional dentistry. With the strong evidence base linking diabetes and periodontal health ${ }^{27}$ along with the aforementioned link with smoking ${ }^{28}$ strengthen their argument for a wider approach to address problems.

The above again reinforces the idea that it is not periodontal disease that is the public health problem. The problem is in the mindset of those designing solutions to problems based on definitions of disease and the adopted care approaches to date. These need to change.

\section{WHERE DOES THAT LEAVE US: THE QUESTIONS THAT NEED ADDRESSING}

It is remarkable that despite the considerable resources allocated within healthcare systems to address what is termed periodontal disease there remains no clear understanding of it or its management. As Prato et al. ${ }^{29}$ comment: 'even today, in periodontology, clinical significance is judged only on the basis of statistical values (for example, the $p$ value).'

This ignores the very purpose of sound periodontal tissues, namely to allow a functional and aesthetic dentition that meets the needs of citizens.

It is not periodontal disease that is the public health problem but the current approach adopted in attempting to address citizens' needs. The approach is flawed. There is a narrowness in the thinking behind the definition and assessment of an individual's needs derived from periodontal measurement. Current models concentrate on the clinical disease and the assessment is defined in ways that are continually changing. The impacts of the range of conditions known as periodontal diseases on individuals are not being taken into account. Instruments that measure this and how they change need to be adopted.

There is a lack of an evidence base to underpin current individual interventions. Even for perhaps the most common intervention adopted, dental health education, Watt and Marinho's ${ }^{30}$ conclusions can be summarised by the question 'If dental health education works why then is there a need to reinforce it every year?'

Despite these issues, delivery systems have continued to support such approaches. They have at the same time failed to adopt arrangements that incentivise healthcare professions to use arrangements that have been shown to be effective. Such arrangements recognise the wider public health approaches needed to address the problem. Jurgensen et al. ${ }^{31}$ summarised matters succinctly. They argued that public health research could facilitate integrated disease prevention, enable the development of appropriate oral health systems and build capacity for public health intervention for periodontal health.

\section{SUMMARY}

In attempting to answer the question as to whether periodontal disease is a public health problem, a series of complex issues are raised, answers to which are more intricate than might initially appear. Perhaps the most critical issue centres on how a society defines and hence what it infers by the term 'periodontal disease'. The current emphasis is based on a definition that adopts clinical disease as its measure. This has considerable implications not least of which is that it seeks the solution on a model of care that is flawed. It cannot and will not ever provide a solution to 'periodontal disease'. This is the public health problem. It is dealing with a challenge that lies in recognising that the periodontal tissues allow a functional dentition that in turn provide individuals with the means to enjoy various qualities of life including eating and smiling.

The more appropriate question lies not in asking whether periodontal disease is a public health problem but whether the approach adopted in the delivery system is. The challenge is to move away from a 'disease centric' model of care to one in which the focus is on the wider qualities associated with health. Such an approach requires health professionals to work with policy makers, the education and training system and politicians to achieve the reorientation of health systems. Periodontal disease may well be associated with a public health problem but the cause lies with the care system itself. The problem with periodontal disease is one of association not causation.
To achieve the goal the care system needs to manage periodontal conditions in terms that move away from one based on a clinical disease model. A focus on aspects such as outcomes of care, 'upstream' health improvements, population orientation and care continuum management that is patient-centred provides an appropriate starting point. ${ }^{32}$ Only then can solutions to the problems arising from the spectrum of periodontal conditions that affect citizens be managed in an efficient and effective manner.

Such a solution does not distract from the role that the dental professions can play in tackling periodontal problems. However, the delivery system needs to adapt to allow care providers to fulfil their potential roles in supporting patients and the public at large. It is a political as well as a clinical role: the professions need to act as advocates for patients. The design of a contract between dental professionals and the State that recognised both the wider determinants of disease and the role that dental professionals could play would be a start. Indeed a contract that concentrated on rewarding outcomes, namely a diminution in treatment need, as opposed to one based simply on the number of interventions, would be a major step forward. Until then periodontal disease will remain a public health problem but it is a consequential not causative link: the current design of the care management system does not help but hinders improvements in disease levels.

1. Paedersen P E. The World Oral Health Report 2003: continuous improvement of oral health in the 21st century - the approach of the WHO Global Oral Health Programme. Community Dent Oral Epidemio 2003; 31 (Suppl 1): 3-24.

2. Daly B, Batchelor P A, Treasure E T, Watt R G. Essential dental public health. 2nd ed. Oxford: Oxford University Press, 2013.

3. Winslow $C A$. The untilled fields of public health. Science 1920; 51: 23-33.

4. Rothstein M A. Rethinking the meaning of public health. J Law Med Ethics 2002; 30: 144-149.

5. Sheiham, A, Watt R. Oral health prevention and policy. 1996. In Murray J J, Nunn J H, Steel J G (eds). pp 241-258. Prevention of oral diseases. 4th ed. Oxford University Press, 2003.

6. Chapple I L C. Time to take periodontitis seriously. BMJ 2014; 348: 2645

7. Petersen P E, Bourgeois D, Ogawa H, Estupinan-Day S, Ndiaye $C$. The global burden of oral diseases and risks to oral health. Bull World Health Organ 2005; 83: 661-669.

8. World Health Organisation. Dental diseases and oral health. 2003. Online information available at http://www.who.int/oral_health/publications/en/ orh_fact_sheet.pdf (accessed 20 July 2014).

9. Chauncey H H, Glass R L, Alman J E. Dental caries. Principal cause of tooth extraction in a sample of US male adults. Caries Res 1989; 23: 200-205.

10. Sheiham A. Public health aspects of periodontal diseases in Europe. J Clin Periodontol 1991; 18: 362-369.

11. Sheiham A, Watt R G. The Common Risk Factor Approach: a rational basis for promoting oral health. Community Dent Oral Epidemiol 2000; 28: 399-406.

12. Watt $R G$, Sheiham $A$. Integrating the common risk factor approach into a social determinants framework. Community Dent Oral Epidemiol 2012; 
40: 289-296.

13. World Health Organisation. Key components of a well functioning health system. 2010. Online information available at http://www.who.int/healthsystems/ EN_HSSkeycomponents.pdf?ua=1 (accessed 15 May 2014).

14. Musgrove P. Public spending on health care: how are different criteria related? Health Policy 1999; 47: 207-223.

15. Musgrove P. Public and private roles in health: theory and financing patterns. World Bank Discussion Paper No. 339. Washington, DC: The World Bank, 1996.

16. Rose G, Khaw K-T, Marmot M. Rose's strategy of preventive medicine.. Oxford: Oxford University Press, 2008.

17. Baelum V, Lopez R Periodontal disease epidemiology - learned and unlearned? Periodontol 2000 2013; 62: 37-58.

18. Borrell L N, Papapanou P N. Analytical epidemiology of periodontitis. J Clin Periodonto/ 2005; 32 (Suppl 6): $132-158$

19. Herrera D, Sanz M, Jepsen S, Needleman I, Roldan S.
A systematic review on the effect of systemic antimicrobials as an adjunct to scaling and root planing in periodontitis patients. J Clin Periodonto/ 2002; 29 (Suppl 3): 136-159.

20. Needleman I, Suvan J, Moles D R, Pimlott J. A systematic review of professional mechanical plaque removal for prevention of periodontal diseases. J Clin Periodontol 2005; 32 (Suppl 6): 229-282.

21. Beirne P V, Worthington H V, Clarkson J E. Routine scale and polish for periodontal health in adults. Cochrane Database Syst Rev 2007: CD004625.

22. Sheiham A, Netuveli G S Periodontal disease in Europe. Periodontol 2000 2002; 29: 104-121.

23. Hugoson A, Norderyd 0 . Has the prevalence of periodontitis changed during the last 30 years? J Clin Periodontol 2008; 35 (Suppl 8): 338-345.

24. Watt R G, Petersen P E. Periodontal health through public health - the case for oral health promotion. Periodontol 2000; 2012: 60: 147-155.

25. Thomson W M, Sheiham A, Spencer A J. Sociobehavioural aspects of periodontal disease. Periodontol 2000 2012; 60: 54-63.
26. Lopez R, Baelum V. Contesting conventional periodontal wisdom: implications for periodontal classifications. Community Dent Oral Epidemiol 2012; 40: 385-395.

27. Kinane D F, Chestnutt I G. Smoking and periodontal disease. Crit Rev Oral Biol Med 2000; 11: 356-365.

28. Preshaw $P$ M, Alba A, Herrera D et al. Periodontitis and diabetes: a two-way relationship. Diabetologia 2012; 55: 21-31.

29. Prato G P, Pagliarob U, Butic J, Rotundod R, Newmane M G. Evaluation of the literature: evidence assessment tools for clinicians J Evid Base Dent Pract 2013; 13: 130-141.

30. Watt R G, Marinho V C. Does oral health promotion improve oral hygiene and gingival health? Periodontol 2000; 2005: 37: 35-47.

31. Jurgensen $N$, Petersen $P E$, Ogawa $H$, Matsumoto $S$. Translating science into action: periodontal health through public health approaches Periodontol 2000 2012; 60: 173-187.

32. Catford J. Turn, turn, turn: time to reorient health services. Health Promot Int 2014; 29: 1-4. 